\title{
Theory and Methodology
}

\section{Opportunity-based block replacement}

\author{
Rommert Dekker * and Eric Smeitink ** \\ Koninklijke/Shell-Laboratorium, Amsterdam P.O. Box 3003, 1003 AA Amsterdam, Netherlands
}

Received January 1989; revised November 1989

\begin{abstract}
In this paper we consider a block replacement model in which a component can be replaced preventively at maintenance opportunities only. Maintenance opportunities occur randomly and are modelled through a renewal process. In the first, theoretical part of the paper we derive an optimality equation and show that the optimal opportunity block replacement policy can be described as a so-called one-opportunity-look-ahead policy. In the second, computational part we present an exact optimisation algorithm in case of $\mathrm{K}_{2}$-distributed times between opportunities. This algorithm can also be used as an approximative method in case of other times between opportunity distributions. Together with another approximative method, based on the stationary forward recurrence time distribution, its performance is checked with simulation.
\end{abstract}

Keywords: Maintenance, optimisation, stochastic processes, probability, phase type distributions

\section{Introduction}

Preventive maintenance is widely accepted within industry as an effective means to reduce the number of failures. Preferably, it is planned at those moments in time when units are not required for production. In the process industry this may cause problems as most units are used continuously and downtime costs are high. Sometimes, however, there may be shortlasting interruptions of production for a variety of reasons, e.g. breakdowns of essential units. During these interruptions some other units are not required and these can then be maintained preventively without costs for downtime being incurred, in which case we speak of maintenance opportunities. Unfortunately, in most cases these opportunities cannot be predicted in advance.

Because of their random occurrence, traditional planning fails to make effective use of these maintenance opportunities. Within the Koninklijke/Shell-Laboratorium, Amsterdam a decision support system has been developed for opportunity maintenance, which is now being field-tested. At the occurrence of an opportunity, the system aids the maintenance engineer in selecting, and assigning priorities to, preventive maintenance activities so as to minimise long-term total average costs.

In this paper we deal with one of the underlying models, viz. the opportunity block replacement model. In this model, a component is replaced upon failure and can only be replaced preventively at an opportunity. The occurrence of opportunities is described by a renewal process. The preventive replace-

* Present address: Shell International Petroleum Maatschappij B.V., P.O. Box 162, 2501 AN The Hague, Netherlands

** Present address: Faculty of Economy and Econometrics, Free University, P.O. Box 7161, 1007 MC Amsterdam, Netherlands. 
ment policies considered prescribe that a component be replaced preventively at the first opportunity after a critical time since the last preventive replacement.

This model was applied for units consisting of many components which were replaced individually upon failure and for which opportunities were created by causes outside the unit (e.g. breakdown of other essential units in a series configuration with the unit). Failures of components provided no maintenance opportunity for other components in the unit as the failure cause had to be removed as soon as possible and there was no time for preventive maintenance on that unit.

The simple age and block replacement model has been widely studied (see e.g. Barlow and Proschan [3]). Models for opportunity (or opportunistic) replacement, however, are scarce. Early work is reported in the review of Pierskalla and Voelker [14] and the book of Jorgenson et al. [11]. More recent work is discussed in the review of Sherif and Smith [17] and in the studies by Sherif [18] and Bäckert and Rippin [2].

Although there are few papers, they present several types of opportunity models. A number of papers consider multiple components and assume that failures of some components create opportunities for preventive maintenance of others. Our model can be regarded as a special case of these models. Discrete-time Markov decision chains are often used to analyse these models, but the computational effort is only bearable in case of few discrete lifetimes and few components. Besides, optimal policies tend to have complex structures and application of these models to our problem yields inferior results compared to our direct approach. A continuous time approach is given in [4]. The solution of the differential equations involved in his approach is only possible in case of special lifetime distributions, such as Erlang distributions.

A model in which opportunities were generated independently of the components to be maintained was first introduced by Jorgenson et al. [11]. For age replacement and exponential times between opportunities they provided formulas for operating characteristics, such as the average costs. For this case Woodman [22] and Duncan and Scholnick [8] provide some numerical results. Sethi [16] considered generally discrete-distributed lifetimes with Markov decision chains and showed that there exists an optimal policy of the control-limit type (a control-limit policy prescribes that a component be replaced at an opportunity if its age has passed a certain critical value). However, he does not show how to determine such a policy. Age replacement at deterministic times between opportunities was incorporated in a model analysed by Berg and Epstein [6].

In our model the opportunity generating process is separated from the component lifetime process, and in case of block replacement this allows a more general and elegant analysis, because the preventive maintenance action only depends on the opportunity process and not on realisations of the lifetimes. It further allows a far better insight into the effect of characteristics of the opportunity process on the optimal policy and the minimum average costs. Although in principle age replacement is a better policy than block replacement, it does not allow such a nice analysis as for block replacement, because at any failure one has to keep track of the residual time to the next opportunity, which has a simple form only in case of exponentially distributed times between opportunities. The opportunity age replacement model is therefore far more difficult to analyse in case of non-exponentially distributed times between opportunities. The exponential case will be dealt with in a subsequent paper (see Dekker and Dijkstra [7]). A further disadvantage of age replacement is that extensions to replacement of multiple components are very difficult, which is not the case for block replacement.

The only paper that has dealt with opportunity block replacement so far is from Liang [12], (he uses the term piggyback policies) whose analysis is only a first step as he provides formulas for operating characteristics (such as average costs) in case of zero control limits only.

In this paper we give theoretical as well as computational results for general continuously distributed lifetimes and times between opportunities. In the theoretical part we focus on establishing an optimality equation without restrictive assumptions and provide an interpretation. In the computational part we present for a special class of opportunity distributions (including the exponential) an exact optimisation algorithm and show how this method can be used as an approximative method for general distributions. Simulation studies are carried out to check the approximations. 


\section{The Opportunity Block Replacement Problem (OBRP)}

Consider a component which may be replaced preventively at an opportunity only against costs $c_{\mathrm{p}}$ $(>0)$. Failure of the component with successive replacement induces costs $c_{\mathrm{f}}\left(c_{\mathrm{f}}>c_{\mathrm{p}}\right)$. Replacements are considered to occur instantaneously. The lifetimes of the components used for replacement are independent and identically distributed; they are represented by the continuous r.v. $X$ with (finite) expectation $\mu$ and variance $\sigma^{2}$. Let $F(t), f(t), M(t)$ and $m(t)$ denote the corresponding cumulative distribution function (c.d.f.), probability density function (p.d.f.), renewal function and renewal density function, respectively. Opportunities occur according to a renewal process, independently of the lifetime process. Let the continuous r.v. $Y$ denote the time between opportunities (abbreviated to TBO). We assume that $Y$ has finite first and second moments and by $G(t), g(t), N(t)$, and $n(t)$ we denote its corresponding c.d.f., p.d.f., renewal function and renewal density function, respectively. We will further assume that both $F(t)$ and $G(t)$ are twice continuously differentiable and that $F(0)=G(0)=0$. As a result both $M(t)$ and $N(t)$ are twice continuously differentiable. For the renewal density $m(t)$ and the renewal function $M(t)$ we have the following asymptotic expressions (see Ross [15] and Tijms [21]). Similar expressions hold for $n(t)$ and $N(t)$.

$$
\lim _{t \rightarrow \infty} m(t)=\frac{1}{\mu}
$$

and

$$
\lim _{t \rightarrow \infty}\left(M(t)-\frac{t}{\mu}\right)=\frac{\sigma^{2}}{2 \mu^{2}}-\frac{1}{2}
$$

We will first state some results from the literature (see e.g. Barlow and Proschan [3] and Berg [5]) on the block replacement problem (abbreviated to BRP). That problem can be considered to be an extreme case of the OBRP by setting the time between opportunities equal to zero (and keeping the cost figures the same). We will refer to this case as the planned case, as it is possible to plan the preventive replacements in advance. In the planned case the process is renewed after each preventive replacement. According to the renewal reward theorem the long-term average costs $\Phi_{\mathrm{p}}(t)$ associated with a replacement interval of length $t$ are given by

$$
\Phi_{\mathrm{p}}(t)=\frac{c_{\mathrm{p}}+c_{\mathrm{f}} M(t)}{t}, \quad t>0
$$

Differentiating (3) with respect to $t$ and rewriting $\Phi_{\mathrm{p}}^{\prime}(t)=0$ yields the equation which the optimal block replacement interval $t_{\mathrm{p}}^{*}$ has to satisfy, i.e.

$$
\operatorname{tm}(t)-M(t)=c_{\mathrm{p}} / c_{\mathrm{f}} .
$$

Furthermore, the minimum expected long-term average cost $\Phi_{\mathrm{p}}^{*}$ (if a solution of (4) exists) equals

$$
\Phi_{\mathrm{p}}^{*}=\Phi_{\mathrm{p}}\left(t_{\mathrm{p}}^{*}\right)=c_{\mathrm{f}} m\left(t_{\mathrm{p}}^{*}\right) .
$$

The marginal cost $\eta_{\mathrm{p}}(t)$ of a preventive replacement at time $t$ (or more precisely, $t$ units of time after the preceding preventive replacement) is defined as the difference, per unit time, between a preventive replacement now and the expected cost associated with waiting an additional infinitesimally short time $\Delta$. More precisely,

$$
\eta_{\mathrm{p}}(t)=\lim _{\Delta \rightarrow 0} \frac{\left(c_{\mathrm{f}}(m(t) \Delta+\mathrm{o}(\Delta))+c_{\mathrm{p}}\right)-c_{\mathrm{p}}}{\Delta}=c_{\mathrm{f}} m(t),
$$

since $m(t) \Delta$ is the expected number of failures in $(t, t+\Delta)$, given only (failures are not recorded) that a 
new component was introduced at time 0 . The equation which $t_{\mathrm{p}}^{*}$ should satisfy is therefore

$$
\eta_{\mathrm{p}}(t)=\Phi_{\mathrm{p}}(t)
$$

which is equivalent to (5) and which we will refer to as the optimality equation for the planned case.

The following theorem from [5] gives sufficient conditions under which a unique, finite solution $t_{\mathrm{p}}^{*}$ exists.

Theorem 1. If $m(t)$ is a continuously increasing function of $t$ then equation (5) has a unique finite solution $t_{\mathrm{p}}^{*}$, provided that

$$
\frac{c_{p}}{c_{f}}<\frac{1}{2}\left(1-\frac{\sigma^{2}}{\mu^{2}}\right)
$$

In the following we will generalize the aforementioned theory and especially the marginal cost approach from [5] to the opportunity block replacement model. Under an opportunity block replacement policy with control limit $t$ a component is replaced preventively at the first opportunity which is at least $t$ time units after the last preventive replacement. It is easily observed that preventive replacements at opportunities are renewals of the total process (both opportunity and lifetime). The process between two successive renewals will be called a cycle. The length of a cycle and the expected number of failures during a cycle depend on the control limit $t$ and the (distribution of the) time between $t$ and the first opportunity after $t$. Let $Z_{t}$ be the random variable denoting the time between $t$ and the first opportunity after $t$ (the forward recurrence time) and let $\Psi(t, \cdot)$ be its c.d.f. Let us first recall some results from renewal theory which can be found in e.g. [15], pp. 44-45. With respect to the distribution of the forward recurrence time it can be shown that

$$
P\left(Z_{t} \leqslant z\right)=G(t+z)-\int_{0}^{t}\{1-G(t+z-u)\} \mathrm{d} N(u), \quad t \geqslant 0, \quad z \geqslant 0,
$$

and

$$
\mathrm{E} Z_{t}=\mathrm{E} Y\{1+N(t)\}-t, \quad t \geqslant 0
$$

Furthermore, there exists a limiting forward recurrence time distribution for which the following equation holds:

$$
P(Z \leqslant z)=\lim _{t \rightarrow \infty} P\left(Z_{t} \leqslant z\right)=\frac{1}{\mathrm{E} Y} \int_{0}^{z}\{1-G(u)\} \mathrm{d} u, \quad z \geq 0
$$

Now we are able to give an expression for the long-term average costs $\Phi_{Y}(t)$ for an opportunity block replacement policy with control limit $t$

$$
\Phi_{Y}(t)=\left(c_{\mathrm{p}}+c_{\mathrm{f}} \int_{0}^{\infty} M(t+z) \mathrm{d} H\left(Z_{t} \leqslant z\right)\right) /\left(t+\mathrm{E} Z_{t}\right)
$$

Notice that the finiteness of $\mathrm{E} Z_{l}$ and the fact that $M(t)$ permits majoration by a linear function (which follows from (2)) guarantee the finiteness of the integral in (12) and that

$$
\lim _{z \rightarrow \infty} M(t+z)\left\{1-P\left(Z_{t} \leqslant z\right)\right\}=0 \quad \text { for every } t \geqslant 0 .
$$

Hence, we can rewrite the integral in (12) with partial integration in the following way

$$
\int_{0}^{\infty} M(t+z) \mathrm{d} P\left(Z_{t} \leqslant z\right)=M(t)+\int_{0}^{\infty} m(t+z)\left\{1-P\left(Z_{t} \leqslant z\right)\right\} \mathrm{d} z
$$

In order to optimise $\Phi_{Y}(t)$ we require the following technical lemma which mainly concerns the change of 
order of differentiation and integration of the integral on the right-hand side of (14). Its proof is given in Appendix 1.

Lemma 2. Let $\Lambda(t) \equiv \int_{0}^{\infty} m(t+z)\left\{1-P\left(Z_{t} \leqslant z\right)\right\} \mathrm{d} z$. Then $\Lambda(t)$ is differentiable in $t$ and

$$
\begin{aligned}
\Lambda^{\prime}(t) & =\int_{0}^{\infty} \frac{\mathrm{d}}{\mathrm{d} t}\left[m(t+z)\left\{1-P\left(Z_{t} \leqslant z\right)\right\}\right] \mathrm{d} z \\
& =-m(t)+E Y n(t) \int_{0}^{\infty} m(t+z) \mathrm{d} P(Z \leqslant z) .
\end{aligned}
$$

Notice that the derivative of $\Lambda(t)$ depends only upon $Z$ and not on $Z_{t}$. Let

$$
\eta_{Y}(t) \equiv c_{r} \int_{0}^{\infty} m(t+z) \mathrm{d} P(Z \leqslant z),
$$

and consider the following equation:

$$
\eta_{Y}(t)-\Phi_{Y}(t)=0
$$

We are now in a position to formulate the main theorem of opportunity block replacement and show that (17) is an optimality equation. The theorem is a direct generalisation of Theorem 1.

Theorem 3. If $m(t)$ is a continuously increasing function, then $\Phi_{Y}(t)$ has its minimum at the unique finite solution $t_{Y}^{*}>0$ of $(17)$, provided that

$$
\frac{c_{p}}{c_{\mathrm{f}}}<\frac{1}{2}\left(1-\frac{\sigma^{2}}{\mu^{2}}\right)
$$

and $n(t)>0$ for $t>0$.

Proof. From (9), (11), (12), (14), (16) and the fact that $M(0)=0$ and $Z_{0}=Y$ it follows that

$$
\Phi_{Y}(0)=\frac{c_{\mathrm{p}}}{\mathrm{E} Y}+\frac{c_{\mathrm{f}}}{\mathrm{E} Y} \int_{0}^{\infty} m(z)\{1-G(z)\} \mathrm{d} z=\frac{c_{\mathrm{p}}}{\mathrm{E} Y}+\eta_{Y}(0)>\eta_{Y}(0) .
$$

Differentiating (12) with respect to $t$ and using (10) and Lemma 2 yields after some algebra

$$
\frac{\mathrm{d}}{\mathrm{d} t} \Phi_{Y}(t)=n(t)\left\{\eta_{Y}(t)-\Phi_{Y}(t)\right\} /(1+N(t))
$$

With $n(t)>0$ for $t>0$ and the continuity of $\Phi_{Y}(t)$ and $\eta_{Y}(t)$ it follows that there is a $\delta>0$ such that $\Phi_{Y}(t)$ is decreasing in the interval $(0, \delta)$. Therefore $t=0$ cannot be a minimum point of $\Phi_{Y}(t)$. From (2) and condition (18) it follows that $\Phi_{Y}(t) \uparrow c_{\mathrm{f}} / \mu$ as $t \rightarrow \infty$. Hence $\Phi_{Y}(t)$ has a minimum. With $\Phi_{Y}(0)>\eta_{Y}(0)$, $n(t)>0$ for $t>0$, the continuity of $\Phi_{Y}(t)$ and $\eta_{Y}(t)$ it follows from (19), that $\Phi_{Y}(t)$ and $\eta_{Y}(t)$ intersect at all extremum points of $\Phi_{Y}(t)$ in such a way that $\eta_{Y}(t)$ crosses from below (above) at the minima (maxima). Hence the equation $\Phi_{Y}(t)=\eta_{Y}(t)$ has at least one finite solution $t_{Y}^{*}>0$, which is a minimum point of $\Phi_{Y}(t)$. This solution is unique, because if $\eta_{Y}(t)$ had intersected $\Phi_{Y}(t)$ once more it must have been at a maximum point of $\Phi_{Y}(t)$ and hence $\Phi_{Y}(t)$ must have been crossed by $\eta_{Y}(t)$ from above - a contradiction with the fact that $m(t)$ and hence $\eta_{Y}(t)$ are increasing functions.

Figure 1 shows two typical examples of the graphs of $\Phi_{Y}(t)$ and $\eta_{Y}(t)$. In Figure 1(a) condition (18) of Theorem 3 is satisfied and there exists a minimum while in Figure 1(b) condition (18) does not hold and there is no minimum. 


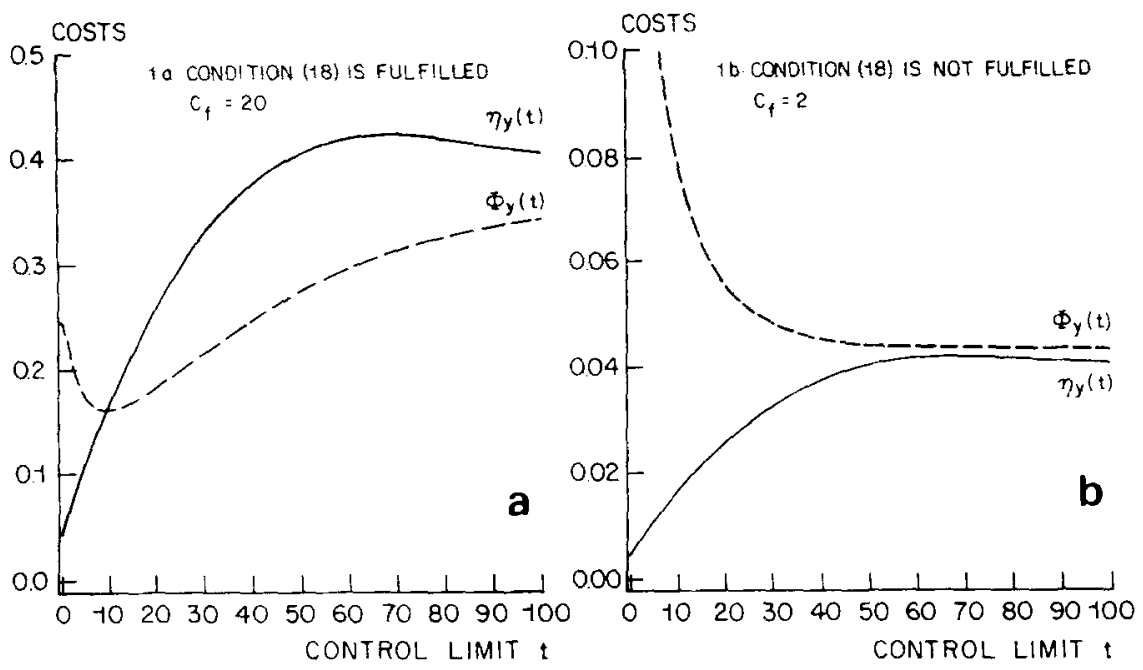

Figure 1. Graphs of average costs $\Phi_{y}(t)$ and marginal costs $\eta_{y}(t)$ (Lifetime distribution: Weibull, mean 50, shape 2; Opportunity distribution: Erlang -2 , mean 5; costs: $c_{\mathrm{p}}=1$ )

Remark 1. In Figure 1 it is seen that $m(t)$ is not increasing for all values of $t$, so strictly speaking Theorem 3 cannot be applied. However, this is merely a theoretical and not a practical problem. The same problem occurs in the BRP. Hanscom and Cleroux [10] show (numerically) that the renewal function $M(t)$ for gamma and Weibull distributions oscillates around the oblique asymptote given by (2). Hence, in general, $m(t)$ is not an increasing function. For the BRP with one component, Hanscom and Cleroux [10] show (again numerically) that if (8) holds, $\Phi_{\mathrm{p}}(t)$ has its absolute minimum in its first local minimum, i.e. at the first point where $\Phi_{\mathrm{p}}(t)$ and $\eta_{\mathrm{p}}(t)$ intersect. This can be extended to the OBRP, i.e. $\Phi_{\gamma}(t)$ has its absolute minimum at the first point where $\Phi_{Y}(t)$ and $\eta_{Y}(t)$ intersect.

We will now compare the planned case with the opportunity case. Notice first that the conditions on the lifetime distribution and cost parameters required for Theorem 3 are the same as for Theorem 1 . This can be understood as follows. Suppose there exists a minimum $t_{\mathrm{p}}^{*}$ in the planned case and that $\Phi_{\mathrm{p}}^{*}<\Phi_{\mathrm{p}}(t)<$ $\mathrm{c}_{\mathrm{r}} / \mu$ for $t>t_{\mathrm{p}}^{*}$. Hence using $t_{\mathrm{p}}^{*}$ as control limit in the opportunity case would lead to average costs lower than $c_{\mathrm{f}} / \mu$ and therefore a minimum has to exist. Intuitively it is also clear that $t_{\mathrm{p}}^{*}>t_{Y}^{*}$ for every $Y$, because even in the opportunity case one will always make use of an opportunity which occurs exactly at $t_{\mathrm{p}}^{*}$. It is however difficult to give a formal proof. In case $Y$ is exponentially distributed (and therefore $Z$, as well) we were able to give the following proof.

Theorem 5. Suppose that the requirements of Theorem 3 are fulfilled and that $Y$ is exponentially distributed, then we have $t_{Y}^{*}<t_{\mathrm{p}}^{*}$.

Proof. First notice that if $Y$ is exponentially distributed then both $Y, Z$ and $Z$, have the same distribution. Secondly, it follows from Theorem 3 that we only need to show that

$$
\eta_{Y}(t)-\Phi_{Y}(t)>0 \text { for } t \geqslant t_{\mathrm{p}}^{*}
$$

Notice that by applying partial integration we can rewrite $\eta_{Y}(t)$ into

$$
\eta_{Y}(t)=\frac{c_{\mathrm{f}}}{\mathrm{E} Y} \int_{0}^{\infty}\{M(t+y)-M(t)\} \mathrm{d} P(Y \leqslant y)
$$


Combining the foregoing yields

$$
\begin{aligned}
\eta_{Y}(t)-\Phi_{Y}(t) & =\int_{0}^{\infty} \frac{c_{\mathrm{f}}\{M(t+y)-M(t)\}}{\mathrm{E} Y}-\frac{c_{\mathrm{p}}+c_{\mathrm{f}} M(t+y)}{t+\mathrm{E} Y} \mathrm{~d} P(Y \leqslant y) \\
& =\int_{0}^{\infty} \frac{c_{\mathrm{f}} t M(t+y)}{\mathrm{E} Y(t+\mathrm{E} Y)}-\frac{c_{\mathrm{f}} M(t)}{\overline{\mathrm{E} Y}}-\frac{c_{\mathrm{p}}}{t+\mathrm{E} Y} \mathrm{~d} P(Y \leqslant y) .
\end{aligned}
$$

For $t \geqslant t_{\mathrm{p}}^{*}$ we have

$$
\frac{c_{\mathrm{p}}+c_{\mathrm{f}} M(t+y)}{t+y}>\frac{c_{\mathrm{p}}+c_{\mathrm{f}} M(t)}{t} \text { for all } y>0 .
$$

Hence,

$$
c_{\mathrm{f}} t M(t+y)>y c_{\mathrm{p}}+(t+y) c_{\mathrm{f}} M(t), \quad t \geqslant t_{\mathrm{p}}^{*}, y>0 .
$$

Inserting this into (21) yields after some algebra

$$
\eta_{Y}(t)-\Phi_{Y}(t)>\int_{0}^{\infty} \frac{y-\mathrm{E} Y}{\mathrm{E} Y(t+\mathrm{E} Y)}\left[c_{\mathrm{p}}+c_{\mathrm{f}} M(t)\right] \mathrm{d} P(Y \leqslant y)=0,
$$

which completes the proof.

\section{The one-opportunity-look-ahead strategy}

In Section 2 we obtained the equation of optimality, (17), for the OBRP by minimising the expected cost per unit time, $\Phi_{Y}(t)$, associated with the control limit policy $t$. We will now show that (17) has an intrinsic meaning, comparable with the marginal cost notion $\eta_{\mathrm{p}}(t)$ in the BRP as introduced in [5]. 2):

Suppose that there is an opportunity at time $t$. We consider the following two alternatives (see Figure

(1) replace the component preventively at this opportunity;

(2) replace the component preventively at the next opportunity $Y$ time units away.

Comparison of these alternatives is not straightforward, since under alternative (1) the process has a renewal at time $t$ and under alternative (2) at time $t+Y$. Notice that the extra expected costs of deferring replacement from the present to the next opportunity are given by the following expression:

$$
c_{\mathrm{f}} \int_{0}^{\infty}\{M(t+y)-M(t)\} \mathrm{d} P(Y \leqslant y),
$$

which is according to (20) equal to $\eta_{Y}(t) \mathrm{E} Y$. Now we define the one opportunity look ahead policy $\pi(\phi)$ with threshold value $\phi$ by:

"replace the component preventively at an opportunity at time $t$ if

$$
\eta_{Y}(t)-\phi \geqslant 0 ”
$$

Such a policy is determined by the choice of $\phi$. Notice that if $m(t)$ is strictly increasing, the one-opportunity look-ahead policy is equal to a control limit policy. Let $\Phi_{Y}^{*} \equiv \min \left\{\Phi_{Y}(t), t>0\right\}$. From Theorem 3 it follows that the optimal control limit policy with control limit $t_{Y}^{*}$ is equivalent with the

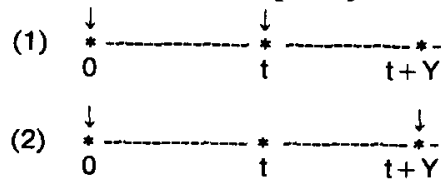

Figure 2. Two alternatives. * indicates an opportunity and $\downarrow$ indicates a preventive replacement (renewal) 
one-opportunity-look-ahead policy with threshold value $\Phi_{Y}^{*}$. In order words, at each opportunity the extra expected costs of deferring preventive replacement to the next opportunity, $\eta_{Y}(t) \mathrm{E} Y$, are compared with the minimum average costs, $\Phi_{Y}^{*}$, times EY. Let $\Phi_{Y}(\pi(\phi))$ denote the average costs under the one-opportunity-look-ahead policy $\pi(\phi)$ with threshold value $\phi$. We have the following theorem

Theorem 6. If $m(t)$ is strictly increasing in $t$ and condition (18) holds, then for every $\phi$ for which $\phi_{Y}^{*} \leqslant \phi<c_{\mathrm{f}} / \mu$ we have

$$
\Phi_{Y}(\pi(\phi)) \leqslant \phi,
$$

with equality only if $\phi=\Phi_{r}^{*}$.

Proof. For $t_{Y}^{*}<t<\infty$ we have $\eta_{Y}(t)>\Phi_{Y}(t)$ and $\lim _{t \rightarrow \infty} \eta_{Y}(t)=\mathrm{c}_{\mathrm{f}} / \mu$. Hence for any $\phi$ satisfying $\Phi_{Y}^{*} \leqslant \phi<c_{\mathrm{f}} / \mu$ there exists a $t_{1}>t_{Y}^{*}$ with $\eta_{Y}\left(t_{1}\right)=\phi$ if $\phi \neq \Phi_{Y}^{*}$ and $t_{1}=t_{Y}^{*}$ if $\phi=\Phi_{Y}^{*}$. Hence $\pi(\phi)$ is equivalent to a control limit policy with control limit $t_{1}$ and $\Phi_{Y}(\pi(\phi))=\Phi_{Y}\left(t_{1}\right)$ from which the assertion directly follows.

Remark 2. In most cases the curve of $\eta_{Y}(t)$ crosses the curve of $\Phi_{Y}(t)$ in its minimum at a rather large angle (e.g. $70^{\circ}$ in figure 1(a)). Accordingly a small error made in calculating $\Phi_{Y}^{*}$ is weakened by using the one-opportunity-look-ahead-policy, as also Theorem 6 states. If in the example of Figure 1(a), we would use $\phi=0.215 \approx \Phi_{Y}(30)$ (instead of $\left.\Phi_{Y}\left(t_{Y}^{*}\right)=\Phi_{Y}(10) \approx 0.161\right)$ then the resulting one-opportunity-lookahead policy is equivalent to a control limit policy with control limit $t^{\prime} \approx 15\left(\eta_{Y}(15) \approx 0.212\right)$ and associated expected long term average costs $\Phi_{Y}(15)=0.168 \approx \Phi_{Y}\left(t_{Y}^{*}\right)$.

\section{Computational aspects}

In this section we present methods to determine the optimal control limit $t_{Y}^{*}$ for the OBRP and the associated expected long-term average cost, i.e. the optimal threshold value $\Phi_{Y}^{*}=\Phi_{Y}\left(t_{Y}^{*}\right)$. Our aim is to develop fast and robust methods which can be used in a decision support system. In doing so we want to use insensitivities and approximations provided that no substantial errors are introduced. The optimal policy $t_{Y}^{*}$ can be obtained by solving the equation of optimality (17) (see also Theorem 3). This can be done in a numerically very efficient and simple way with a bisection or regula falsi method. It only requires an interval $\left[t_{a}, t_{b}\right]$ that contains the unique solution $t_{Y}^{*}$ and the functions $\phi_{Y}(t)$ and $\eta_{Y}(t)$. From (12) and (16) it is seen that calculation of $\eta_{Y}(t)$ and $\Phi_{Y}(t)$ requires

(1) an algorithm to approximate the renewal function $M(t)$, since in general no analytical expression for $M(t)$ exists;

(2) the distribution function $P\left(Z_{t} \leqslant z\right)$ of the time between $t$ and the first opportunity after $t$ (forward recurrence time) and its expectation;

(3) numerical integration of improper integrals.

We will not go into detail about the numerical integration. Truncating the improper integrals should be done with care because the tail of the distribution of the forward recurrence time may have a large contribution in case of large coefficients of variation. Good algorithms exist to treat improper integrals as the one in (12). We used an algorithm based on Gauss-Laguerre expansion which performed very well (see e.g. [20]).

\subsection{A new method to approximate the renewal function}

For most distributions used in reliability (e.g. the Weibull, gamma, and truncated normal distributions) no analytical expression for the renewal function $M(t)$ exists. There are several methods to approximate the renewal function, e.g. power series expansion and discretisation (see e.g. [9]). Calculating and 
optimising $\Phi_{Y}(t)$ involves repeated calculation of $M(t)$. Using the OBRP in a decision support system therefore requires a very fast and robust approximation for the renewal function. We developed a simple but effective method in which the original distribution is approximated by a phase-type distribution with the same first and second moment, for which the renewal function can be easily computed. We used the $\mathrm{E}_{k-1, k}$ distribution to approximate lifetime distributions with squared coefficient of variation $c_{X}^{2}\left(\equiv \sigma^{2} / \mu^{2}\right)$ $\leqslant \frac{1}{2}$ and the $K_{2}$ distribution (also called Coxian-2 distribution) in case $c_{X}^{2}>\frac{1}{2}$ (see [21, pp. 397-400]). The approximation $M_{1}(t)$ has the following form

$$
M_{1}(t)=F(t)+\sum_{n=2}^{\infty} \hat{F}^{(n)}(t), \quad t \geqslant 0
$$

or equivalently,

$$
M_{1}(t)=\hat{M}(t)+[F(t)-\hat{F}(t)], \quad t \geqslant 0,
$$

where $\hat{F}^{(n)}(t)$ and $\hat{M}(t)$ are the $n$-fold convolution and renewal function of the c.d.f. of the approximating phase-type distribution respectively. It appears that the simple approximation method given above works very well. For Weibull and gamma distributions with $c_{X}^{2} \leqslant 1$ (i.e. with increasing failure rate) the maximum relative error is typically smaller than 3\%. Especially for small values of $t$ (say $0<t<\mu$ ) this approximation is an improvement on ordinary two-moment approximations since the probability on one or more failures in the interval $[0, t]$ is calculated exactly. In determining an optimal block replacement interval this is important, since in most practical situations the optimal replacement interval (if any) will be in this range. Using the approximation in the block replacement problem yields results that are fully satisfactory, i.e. the resulting error in $\Phi_{Y}^{*}$ is smaller that $0.1 \%$. More details on this procedure can be found in [19].

\subsection{The distribution of the forward recurrence time}

In general evaluating $(9)$ in order to obtain $P\left(Z_{t} \leqslant z\right)$ will be difficult. It requires integration of the product of the distribution function $G(\cdot)$ and the associated renewal density $n(\cdot)$, which can only be approximated numerically. Calculation of $\Phi_{Y}(t)$ in turn requires integration over $P\left(Z_{t} \leqslant z\right)$, so it will be clear that we should not use (9) if we want to develop a fast algorithm to calculate $\Phi_{Y}(t)$.

However, for phase-type distributions (see [13, pp. 44-48; 63-70]) there is a simple, explicit expression for $P\left(Z_{t} \leqslant z\right)$ (see Appendix 2). In fact, $Z_{t}$ is also of phase type with the same number of phases, $n$, and its distribution can be calculated by solving a set of $n$ linear differential equations. For the $K_{2}$ distribution, a phase-type distribution with only two phases and probability density function

$$
f(t)= \begin{cases}p \lambda \mathrm{e}^{-\lambda t}+(1-p) \lambda^{2} t \mathrm{e}^{-\lambda t}, & \lambda_{1}=\lambda_{2}=\lambda, \\ \frac{p \lambda_{1}-\lambda_{2}}{\lambda_{1}-\lambda_{2}} \lambda_{1} \mathrm{e}^{-\lambda_{1} t}+\left(1-\frac{p \lambda_{1}-\lambda_{2}}{\lambda_{1}-\lambda_{2}}\right) \lambda_{2} \mathrm{e}^{-\lambda_{2} t}, & \lambda_{1} \neq \lambda_{2},\end{cases}
$$

the solution is easily obtained analytically and is given by

$$
P\left(Z_{t} \leqslant z\right)=\left\{\begin{array}{l}
1-w_{1}(t) \frac{p \lambda_{1}-\lambda_{2}}{\lambda_{1}-\lambda_{2}} \mathrm{e}^{-\lambda_{1} z}-\left[1-w_{1}(t) \frac{p \lambda_{1}-\lambda_{2}}{\lambda_{1}-\lambda_{2}}\right] \mathrm{e}^{-\lambda_{2} z}, \quad \lambda_{1} \neq \lambda_{2}, \\
1-\mathrm{e}^{-\lambda z}-w_{1}(t)(1-p) \lambda z \mathrm{e}^{-\lambda z}, \quad \lambda_{1}=\lambda_{2}=\lambda,
\end{array}\right.
$$

where

$$
w_{1}(t)=\frac{\lambda_{2}}{\lambda_{1}(1-p)+\lambda_{2}}-\frac{\lambda_{1}(1-p)}{\lambda_{1}(1-p)+\lambda_{2}} \mathrm{e}^{-\left\{\lambda_{1}(1-p)+\lambda_{2}\right\} t}
$$



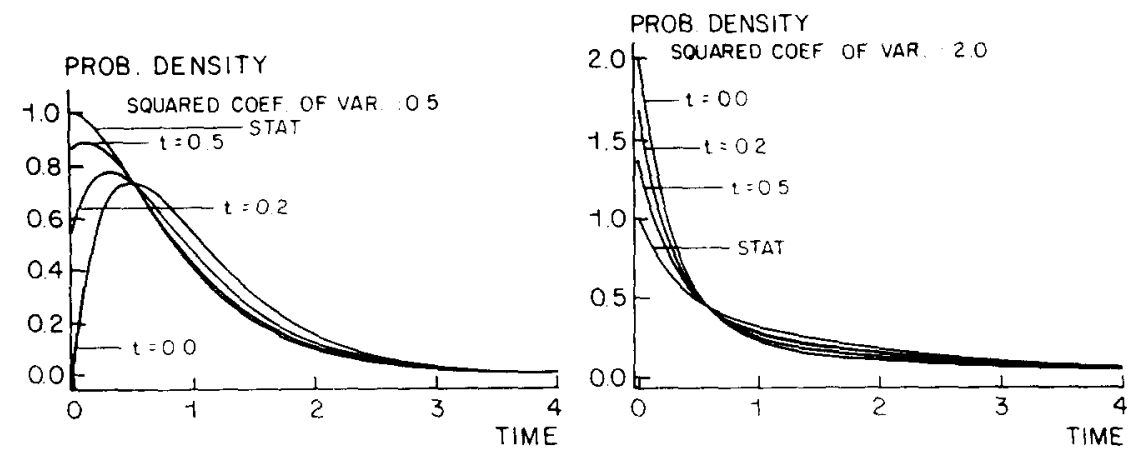

Figure 3. Probability density of the forward recurrence time for the $\mathrm{K}_{2}$ distribution (mean: 1.0 , normalisation: gamma)

For the derivation of this result we refer to Appendix 2. The corresponding probability density of $Z_{t}$, $\psi(t, z)$, and $\mathrm{E} Z$, are easily obtained from (26).

A numerical comparison between the probability densities $\psi(t, z)$ and the probability density of the stationary time between opportunities, $Z, \psi(z)$, shows that especially for moderate values of $c_{Y}^{2}$ (say between $\frac{1}{2}$ and $\left.1 \frac{1}{2}\right) \psi(t, z)$ converges quickly to $\psi(z)$ (see Figure 3). For $t=\frac{1}{2} \mathrm{E} Y \psi(z)$ is already a good approximation to $\psi(t, z)$. This led us to the idea to approximate $\psi(t, z)$ by $\psi(z)$ in the calculation of $\Phi_{Y}(t)$, which we call the stationary approximation. For $\frac{1}{2} \leqslant c_{v}^{2}$ we used the analytical results for the $K_{2}$ distribution to compare the average cost curves obtained with $\psi(t, z)$ and $\psi(z)$ (see Figure 4). In all cases both curves have a minimum for practically the same control limit. It will be clear that the approximation is better for large values of $t$. Hence the stationary approximation will perform well if the optimal control limit is not small compared to $\mathrm{E} Y$.

\subsection{Comparison of approximations with simulation results}

We carried out a simulation study in order to compare the optimal strategy and the strategies obtained by using either the $K_{2}$ or the stationary approximation to the TBO distribution. Also, we investigated the effect of the form of the TBO distribution on the optimal control limit and the minimum average costs.

We considered three unimodal time-between-opportunity distributions, viz. the gamma, Weibull and lognormal distributions, and varied their coefficient of variation $c_{Y}(=\sigma(Y) / E Y)$ from 0.25 to 2 . The values of 1.5 and 2 were only incorporated for academic reasons as the limited data obtained so far on
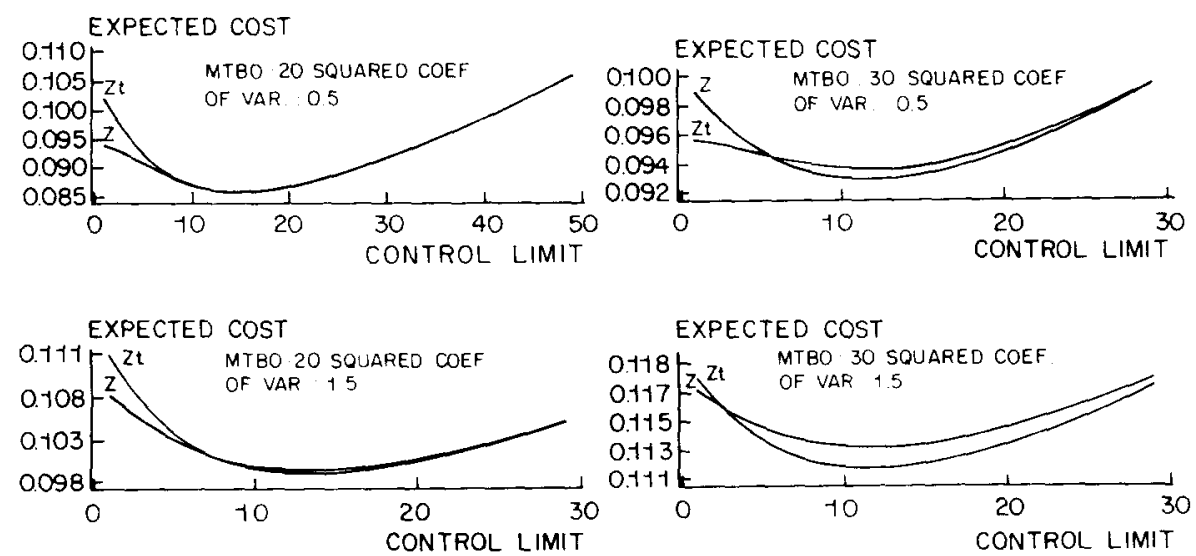

Figure 4. Cost curves for opportunity based block replacement. Comparison integration over stationary/time dependent forward recurrence time for $\mathrm{K}_{2}$ distribution (Failure distribution: Weibull, mean time to failure: 100, cost ratio $c_{\mathrm{f}} / c_{\mathrm{p}}: 20$, shape beta of failure distribution: 2.0 , opportunity distribution: $\left.\mathrm{K}_{2}(\mathrm{MTBE} \equiv \mathrm{EY})\right)$ 
Table 1

Comparison of the different approximations of the distribution of the time between opportunities (component lifetime: Weibull distributed, $\mathrm{E} X=10$, shape $\beta=2$; time between opportunities: Weibull distributed, $\mathrm{E} Y=2$; $\operatorname{costs}: c_{\mathrm{p}}=1, c_{\mathrm{f}}=20$; optimum planned replacement: $t_{\mathrm{p}}^{*}=2.6$ )

\begin{tabular}{|c|c|c|c|c|c|c|}
\hline & \multicolumn{6}{|l|}{$c_{Y}$} \\
\hline & 0.25 & 0.5 & 0.75 & 1.0 & 1.5 & 2.0 \\
\hline $\operatorname{sim} t_{Y}^{*}$ & 1.66 & 1.59 & 1.49 & 1.413 & 1.34 & 1.34 \\
\hline$t_{\mathrm{K}_{2}}^{*}$ & & & 1.493 & 1.413 & 1.352 & 1.384 \\
\hline $\mathrm{t}_{\text {stat }}^{*}$ & 1.670 & 1.574 & 1.504 & 1.413 & 1.604 & 1.938 \\
\hline $\operatorname{sim} . \Phi_{\gamma}^{*}$ & 0.805 & 0.821 & 0.865 & 0.928 & 1.067 & 1.187 \\
\hline $\operatorname{sim} . \Phi_{Y}\left(t_{\mathrm{K}_{2}}^{*}\right)$ & & & 0.865 & 0.928 & 1.067 & 1.187 \\
\hline $\operatorname{sim} . \Phi_{Y}\left(t_{\text {stat }}^{*}\right)$ & 0.805 & 0.821 & 0.865 & 0.928 & 1.070 & 1.198 \\
\hline$\Phi_{\mathrm{K}_{z}}^{*}$ & & & 0.866 & 0.928 & 1.086 & 1.238 \\
\hline$\Phi_{\text {stat }}^{*}$ & 0.806 & 0.824 & 0.863 & 0.928 & 1.115 & 1.302 \\
\hline
\end{tabular}

opportunities showed values of $c_{Y}$ ranging between 0.4 and 0.8 . As component lifetime distribution we used a two-parameter Weibull distribution. It will be clear that the effect of restricting preventive maintenance to opportunities will be small if $\mathrm{E} Y$ is small with respect to $t_{\mathrm{p}}^{*}$, the optimum control limit in the planned case. On the other hand, cases where $E Y$ is much larger than $t_{\mathrm{p}}^{*}$ are not realistic as in that case one would not require optimisation of opportunity maintenance. Therefore we considered a case where $\mathrm{E} Y / \mathrm{E} X=0.2, \mathrm{E} Y / t_{\mathrm{p}}^{*}=0.8$ and $\beta=2$ (see Table 1) and checked the conclusions with a case in which $\mathrm{E} Y / \mathrm{E} X=0.5, \mathrm{E} Y / t_{\mathrm{p}}^{*}=1.25$ and $\beta=4$ (see Table 2).

In Tables 1 and 2 the optimal strategy is compared with the strategies obtained by approximating the TBO distribution (Weibull) with a $\mathrm{K}_{2}$ distribution (two-moment fit and gamma normalisation) or using $\psi(z)$ instead of $\psi(t, z)$ (stationary approximation). Similar calculations were done in case of gamma- and lognormal distributed times between opportunities. In the tables $\mathbf{a}^{*}$ indicates optimality. For example, $t_{\mathbf{K}_{2}}^{*}$ is the optimal control limit in case of a $\mathrm{K}_{2} \mathrm{TBO}$ distribution and $\Phi_{\mathrm{K}_{2}}^{*}$ is the corresponding minimum average cost. When using the stationary approximation the minimum average costs and optimal control limit are denoted by $\Phi_{\text {stat }}^{*}$ and $t_{\text {stat }}^{*}$, respectively. Furthermore, $\Phi_{Y}\left(t_{\mathrm{K}_{2}}^{*}\right)$ is the average cost resulting from using the control limit $t_{\mathrm{K}_{2}}^{*}$ if in fact the time between opportunities, $Y$, is not $\mathrm{K}_{2}$ distributed ('sim.' indicates that results were obtained by simulation).

In Table 3 the optimal strategies for various TBO distributions are compared. Except for the $\mathrm{K}_{2}$-distribution all results of Table 3 are obtained by simulation.

Table 2

Comparison of the different approximations of the distribution of the time between opportunities (component lifetime: Weibull distributed, $\mathrm{E} X=10$, shape $\beta=4$; time between opportunities: Weibull distributed, $\mathrm{E} Y=5$; $\operatorname{costs:} c_{\mathrm{p}}=1, c_{\mathrm{f}}=20$; optimum planned replacement: $t_{\mathrm{p}}^{*}=4.0$ )

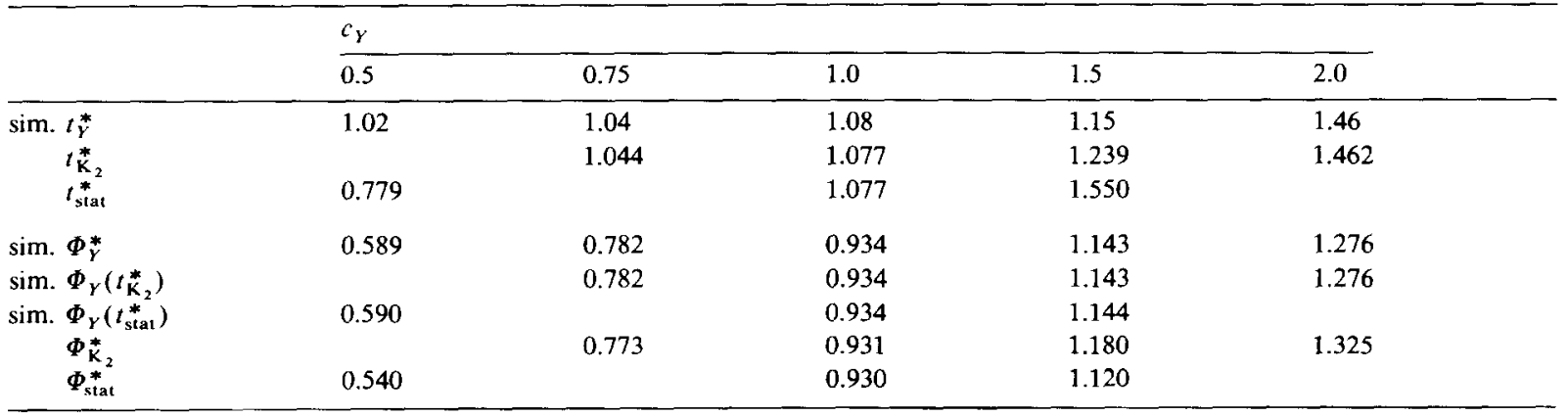


Table 3

Influence of the form of the distribution of the times between opportunities (component lifetime: Weibull distributed, $\mathrm{E} X=10$, shape $\beta=2$; time between opportunities: $\mathrm{E} Y=2$; $\cos t \mathrm{~s}: c_{\mathrm{p}}=1, c_{\mathrm{f}}=20$ )

\begin{tabular}{|c|c|c|c|c|c|c|}
\hline \multicolumn{7}{|l|}{$Y$ distribution } \\
\hline & & \multicolumn{5}{|l|}{$c_{Y}$} \\
\hline & & 0.5 & 0.75 & 1.0 & 1.5 & 2.0 \\
\hline sim. gamma & $t_{Y}^{*}$ & 1.61 & 1.49 & 1.41 & 1.35 & 1.21 \\
\hline sim. Weibull & $t_{Y}^{*}$ & 1.59 & 1.49 & 1.41 & 1.34 & 1.34 \\
\hline sim. lognormal & $t_{Y}^{*}$ & 1.60 & 1.55 & 1.52 & 1.48 & 1.45 \\
\hline $\mathrm{K}_{2}$ & $t_{\mathrm{K}_{2}}^{*}$ & & 1.493 & 1.413 & 1.352 & 1.384 \\
\hline sim. gamma & $\Phi_{Y}^{*}$ & 0.825 & 0.867 & 0.928 & 1.074 & 1.218 \\
\hline sim. Weibull & $\Phi_{Y}^{*}$ & 0.821 & 0.865 & 0.928 & 1.067 & 1.187 \\
\hline sim. lognormal & $\Phi_{\gamma}^{*}$ & 0.829 & 0.874 & 0.928 & 1.033 & 1.115 \\
\hline $\mathrm{K}_{2}$ & $\Phi_{\mathrm{K}_{2}}^{*}$ & & 0.866 & 0.928 & 1.086 & 1.238 \\
\hline
\end{tabular}

Finally, in Table 4 we illustrate the effect of using the optimum control limit for the planned case, $t_{\mathrm{p}}^{*}$, as control limit instead of the actual optimum, $t_{\mathrm{K}_{2}}^{*}$, in case of $\mathrm{K}_{2}$-distributed times between opportunities. This leads to suboptimal strategies with resulting costs that are typically $5 \%$ to $10 \%$ higher than the minimal costs.

The simulation results were obtained as follows. For a fixed control limit $t$, point estimates for the expected number of failures until preventive replacement, $M\left(t+Z_{t}\right)$, were obtained by integrating the life-time renewal function (determined with the method from Section 4.1) over the distribution of $Z_{t}$ using Monte Carlo simulation. In the same manner we obtained point etimates for the expected cycle length $t+\mathrm{E} Z_{t}$. The long term average costs are then readily calculated using (12). The half length of the $95 \%$ confidence intervals was in all cases less than 0.01 .

The optimal control limit was obtained using Theorem 3 (17) and a bisection procedure with stopping criterion

$$
\left|\eta_{Y}(t)-\Phi_{Y}(t)\right| / \Phi_{Y}(t)<1 \%,
$$

where $\eta_{Y}(t)$ was calculated using (20). An excellent starting value for the bisection procedure is the control limit obtained by optimising the average costs using the $\mathrm{K}_{2}$ (or the stationary) approximation.

Table 4

Effect of using the optimal control limit from the planned case (component lifetime: Weibull distributed, E $X=10$, shape $\beta$; time between opportunities: $K_{2}$ distributed; costs: $c_{p}=1, c_{f}=20$ )

\begin{tabular}{llllll}
\hline case & & $c_{Y}$ & & & \\
\cline { 3 - 5 } & & 0.75 & 1.0 & 1.5 & 2.0 \\
\hline $\mathrm{E} Y=2$ & $t_{\mathrm{K}}^{*}$ & 1.493 & 1.413 & 1.352 & 1.384 \\
$\beta=2$ & $\Phi_{\mathrm{K}_{2}}^{*}$ & 0.866 & 0.928 & 1.118 & 1.238 \\
$t_{\mathrm{p}}^{*}=2.6$ & $\Phi_{\mathrm{K}_{2}}\left(t_{\mathrm{p}}^{*}\right)$ & 0.902 & 0.963 & 1.032 & 1.267 \\
$\mathrm{E} Y=5$ & $t_{\mathrm{K}_{2}}^{*}$ & 0.880 & 0.919 & 1.397 & 1.158 \\
$\beta=2$ & $\Phi_{\mathrm{K}_{2}}^{*}$ & 1.133 & 1.232 & 1.425 & 1.496 \\
$t_{\mathrm{p}}^{*}=2.6$ & $\Phi_{\mathrm{K}_{2}\left(t_{\mathrm{P}}^{*}\right)}$ & 1.167 & 1.264 & 1.239 & 1.525 \\
$\mathrm{E} Y=5$ & $t_{\mathrm{K}_{2}}^{*}$ & 1.044 & 1.077 & 1.180 & 1.462 \\
$\beta=4$ & $\Phi_{\mathrm{K}_{2}}^{*}$ & 0.773 & 0.931 & 1.255 & 1.325 \\
$t_{\mathrm{P}}^{*}=4.0$ & $\Phi_{\mathrm{K}_{2}}\left(t_{\mathrm{p}}^{*}\right)$ & 0.893 & 1.033 & 1.386 \\
\hline
\end{tabular}


Conclusions from the tables

(1) With the $\mathrm{K}_{2}$ distribution the forward recurrence time distribution can be satisfactory approximated for a wide class of (continuous and unimodal) distributions (with $c_{Y}^{2}>\frac{1}{2}$ ). For distributions of the time between opportunities with $c_{Y}^{2} \leqslant \frac{1}{2}$ the stationary approximation can be used, provided that the ratio $\mathrm{E} Y / \mathrm{E} X$ is not too large. It is difficult to give a good rule of thumb, but when $\mathrm{E} Y / \mathrm{E} X$ was between 0 and 0.2 the resulting error made by the stationary approximation was very small in our experiments (see Tables 1 and 2).

(2) Our approximations yield computationally tractable results that outperform a simple strategy such as using the optimal control limit $t_{\mathrm{p}}^{*}$ for the planned case (see Table 4).

(3) The minimum average costs $\Phi_{Y}^{*}$ and the optimal control limit $t_{Y}^{*}$ depend substantially on the mean EY and coefficient of variation $c_{Y}$ of the time between opportunities (see Table 1 and 2). $\Phi_{Y}^{*}$ increases with $\mathrm{E} Y$ and $c_{Y}$, while $t_{Y}^{*}$ decreases with $\mathrm{E} Y$ and can either increase or decrease with $c_{Y}$. The type of distribution is of far less importance, especially for low coefficients of variation (see Table 3 ).

(4) The approximation of $\Phi_{Y}^{*}$ by $\Phi_{\mathrm{K}}^{*}$, yields errors less than $2 \%$ for gamma and Weibull distributions. For the lognormal distribution the error remains small $(<5 \%)$ in case $c_{Y}<1.5$. The approximation of $\Phi_{Y}^{*}$ by $\Phi_{\text {stat }}^{*}$ is slightly worse than the approximation by $\Phi_{\mathbf{K}_{2}}^{*}$ but satisfactory for values of $c_{Y}$ between 0.25 and 1.5. Both approximations are best for low values of $\mathrm{E} Y / \mathrm{E} X$ and values of $c_{Y}$ that are close to 1 .

(5) The errors made in approximating $t_{Y}^{*}$ by either $t_{\mathrm{K}_{2}}^{*}$ or $t_{\text {stat }}$ can be large, e.g. up to $50 \%$ for $t_{\text {stat }}$ and $20 \%$ for $t_{\mathrm{K}_{2}}^{*}$ in the (extreme) cases with $c_{Y}=2$. However, as the average cost curves are quite flat around their minimum the error in the average costs resulting from the use of these approximations is much smaller.

\section{Extensions}

There are a number of ways in which the previous theory can be extended. First of all, we will show that block replacement can also be extended to multiple components. Secondly, one-opportunity-look-ahead policies can be used in setting priorities for execution of maintenance packages if only a limited number of these can be executed at a given opportunity. The latter case will be treated in a subsequent paper.

\subsection{Extension of the OBRP to a multicomponent case}

So far we have considered one component only. In practice, maintenance activities involving replacement of components are usually combined into a maintenance package. When executed preventively, always the whole package is carried out, whereas upon failure, only the failed component is replaced. The problem then is to determine the optimal control limit for execution of the maintenance package. The extension of the OBRP model to a multi-component case is straightforward. Notice that the entire analysis of Sections 2 and 3 can be applied. The only change required in the formulas is that we have to distinguish $n$ possibly different components. For example, in the formula for $\Phi_{Y}(t)$ we replace $c_{\mathrm{f}} M(t)$ by

$$
\sum_{i=1}^{n} c_{f}^{(i)} M_{i}(t)
$$

where the index $i$ indicates component $i$. Analogous changes have to be made in the other formulas. All the results remain valid, only in Theorem 3 we require that

$$
c_{\mathrm{p}}<\frac{1}{2} \sum_{i=1}^{n} c_{\mathrm{f}}^{(i)}\left(1-\frac{\sigma_{i}^{2}}{\mu_{i}^{2}}\right)
$$


instead of condition (18), and furthermore that

$$
\sum_{i=1}^{n} c_{f}^{(i)} m_{i}(t)
$$

must be increasing (instead of $m(t)$ ). Similar problems as mentioned in Remark 2 with respect to the increase of $m(t)$ are encountered in the last condition. If the component lifetimes are quite different it poses severe problems as there may be multiple minima and the first minimum does not need to be the absolute minimum.

\section{Appendix 1. Proof of Lemma 2.}

The main difficulty is to show that the order of integration and differentiation may be interchanged. To use a standard theorem of analysis (see e.g. [1]) we have to make the following three observations.

(1) The integral $\int_{0}^{\infty} m(t+z)\{1-\Psi(t, z)\} \mathrm{d} z$ is convergent on [0, $\left.\infty\right)$. This follows from the fact that $m(\cdot)$ allows majoration by a constant and $E Z_{t}$ is finite for every $t \geq 0$. The majoration of $m(\cdot)$ follows from the renewal density theorem and the continuity of $m(\cdot)$.

(2) The function $(\mathrm{d} / \mathrm{d} t) m(t+z)\{1-\Psi(t, z)\}$ is continuous on $[0, \infty) \times[0, \infty)$.

This follows directly from the assumption that $m(\cdot)$ is continuously differentiable and (9) combined with the assumption that $G(\cdot)$ and $n(\cdot)$ are continuously differentiable.

(3) The integral

$$
\int_{0}^{\infty} \frac{\mathrm{d}}{\mathrm{d} t}[m(t+z)\{1-\Psi(t, z)\}] \mathrm{d} z
$$

converges uniformly in $t$ on $[0, \infty)$. To see this we write

$$
\begin{aligned}
\int_{0}^{N} & \frac{\mathrm{d}}{\mathrm{d} t}[m(t+z)\{1-\Psi(t, z)\}] \mathrm{d} z \\
= & \int_{0}^{N}\{1-\Psi(t, z)\} \frac{\mathrm{d}}{\mathrm{d} t} m(t+z) \mathrm{d} z+\int_{0}^{N} m(t+z) \frac{\partial}{\partial t}\{1-\Psi(t, z)\} \mathrm{d} z \\
= & \{1-\Psi(t, N)\} m(t+N)-\{1-\Psi(t, 0)\} m(t) \\
& -\int_{0}^{N} m(t+z) \frac{\partial}{\partial z}\{1-\Psi(t, z)\} \mathrm{d} z+\int_{0}^{N} m(t+z) \frac{\partial}{\partial t}\{1-\Psi(t, z)\} \mathrm{d} z \\
= & \{1-\Psi(t, N)\} m(t+N)-\{1-\Psi(t, 0)\} m(t) \\
& +\int_{0}^{N} m(t+z)\left\{\frac{\partial}{\partial z} \Psi(t, z)-\frac{\partial}{\partial t} \Psi(t, z)\right\} \mathrm{d} z .
\end{aligned}
$$

From (9) it follows that

$$
\frac{\partial}{\partial z} \Psi(t, z)-\frac{\partial}{\partial t} \Psi(t, z)=\{1-G(z)\} n(t) .
$$

Hence, using (31) we can write

$$
\begin{aligned}
& \int_{0}^{N} \frac{\mathrm{d}}{\mathrm{d} t}[m(t+z)\{1-\Psi(t, z)\}] \mathrm{d} z \\
& \quad=\{1-\Psi(t, N)\} m(t+N)-\{1-\Psi(t, 0)\} m(t)+\operatorname{EY} n(t) \int_{0}^{N} m(t+z) \mathrm{d} P(Z \leqslant z) .
\end{aligned}
$$


It is easy to show that $\Psi(t, z)$ converges uniformly to $\Psi(z)$. Now with (32) and the finiteness of EZ it follows that (30) converges uniformly on $[0, \infty)$. Having made these three observations, we can use Theorem 14-24 (p. 443) of [1] to change the order of integration and differentiation and with (32) we obtain, noting that $\Psi(t, 0)=0$,

$$
\begin{aligned}
\frac{\mathrm{d}}{\mathrm{d} t} \int_{0}^{\infty} m(t+z)\{1-\Psi(t, z)\} \mathrm{d} z & =\int_{0}^{\infty} \frac{\mathrm{d}}{\mathrm{d} t}[m(t+z)\{1-\Psi(t, z)\}] \mathrm{d} z \\
& =\operatorname{EYn}(t) \int_{0}^{\infty} m(t \mid z) \mathrm{d} P(Z \leqslant z)
\end{aligned}
$$

which concludes the proof.

\section{Appendix 2}

In this appendix we give definitions of the Weibull and $\mathrm{K}_{2}$ distributions and derive the distribution of the forward recurrence time $Z_{t}, P\left(Z_{t} \leqslant z\right)$ for phase type distributions. For the $\mathrm{K}_{2}$ distribution we derive an analytical expression for $P\left(Z_{t} \leqslant z\right)$.

\section{Definition of the Weibull distribution}

A random variable $X$ has a Weibull distribution with scale parameter $\lambda$ and shape parameter $\beta$ if its probability density function satisfies

$$
f(t)=\left(\frac{t}{\lambda}\right)^{\beta-1} \exp \left\{-\left(\frac{t}{\lambda}\right)^{\beta}\right\}, \quad t \geqslant 0 .
$$

\section{Definition of the $\mathrm{K}_{2}$ distribution}

$\Lambda$ random variable $X$ has a $\mathrm{K}_{2}$ distribution if

$$
X= \begin{cases}X_{1} & \text { with probability } p \\ X_{1}+X_{2} & \text { with probability } 1-p\end{cases}
$$

where $X_{1}$ and $X_{2}$ are independent and exponentially distributed with parameters $\lambda_{1}$ and $\lambda_{2}$, respectively and $0 \leqslant p \leqslant 1, \lambda_{1}>0, \lambda_{2}>0$. The $\mathrm{K}_{2}$ distribution corresponds to the sojourn time (in states 1 or 2 ) in the continuous-time Markov chain of Figure 5. The probability density function of a $\mathbf{K}_{2}$ distribution is given by:

$$
f(t)= \begin{cases}p \lambda \mathrm{e}^{-\lambda t}+(1-p) \lambda^{2} t \mathrm{e}^{-\lambda t}, & \lambda_{1}=\lambda_{2}=\lambda, \\ \frac{p \lambda_{1}-\lambda_{2}}{\lambda_{1}-\lambda_{2}} \lambda_{1} \mathrm{e}^{-\lambda_{1} t}+\left(1-\frac{p \lambda_{1}-\lambda_{2}}{\lambda_{1}-\lambda_{2}}\right) \lambda_{2} \mathrm{e}^{-\lambda_{2} t}, & \lambda_{1} \neq \lambda_{2} .\end{cases}
$$

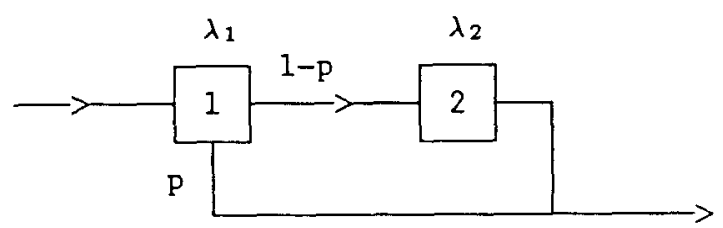

Figure 5. Markov diagram of a $\mathrm{K}_{2}$ distribution 
Fitting a $\mathrm{K}_{2}$ distribution on the first two moments leaves some degree of freedom in the choice of the parameters $\lambda_{1}, \lambda_{2}$ and $p$. In some cases the third moment can also be fitted, but this is not always possible. A good alternative is to use a gamma normalisation to obtain a unique fit. This means that $\lambda_{1}, \lambda_{2}$ and $p$ are chosen in such a way that the third moment of the $\mathrm{K}_{2}$ distribution equals the third moment of a gamma distribution with the same first and second moment as $X$. This is always possible (see also [21, pp. $399-400])$ and $\lambda_{1}, \lambda_{2}$ and $p$ are given by

$$
\lambda_{1}=\frac{2}{\mathrm{E} X}\left(1+\left(\frac{c_{X}^{2}-\frac{1}{2}}{c_{X}^{2}+1}\right)^{1 / 2}\right), \quad \lambda_{2}=\frac{4}{\mathrm{E} X}-\lambda_{1}, \quad p=\left(1-\lambda_{2} \mathrm{E} X\right)+\frac{\lambda_{2}}{\lambda_{1}}
$$

The distribution of the forward recurrence time for PH-distributions

Consider a continuous-time Markov chain with state space $\{1, \ldots, m+1\}$. States $1, \ldots, m$ are transient and state $m+1$ is absorbing. The infinitesimal generator $Q$ of such a Markov chain has the form

$$
Q=\left(\begin{array}{cc}
T & T^{0} \\
0 & 0
\end{array}\right)
$$

where $T$ is a nonsingular $m \times m$ matrix and $T^{0}$ is an $m$-vector. Matrix $T$ has $T_{i i}<0$, for $1 \leqslant i \leqslant m$, and $T_{i j} \geqslant 0$ for $i \neq j$. Vector $T^{0} \geqslant 0$ satisfies $T e+T^{0}=0$ where $e^{\prime}=(1, \ldots, 1)$. Let $\left(\alpha, \alpha_{m+1}\right)$ denote the vector of initial probabilities, where $\alpha$ is an $m$ vector such that $0<\alpha e \leqslant 1$. The distribution $G$ of the time until absorption in state $m+1$ given the initial probability vector $\left(\alpha, \alpha_{m+1}\right)$ is

$$
G(x)=1-\alpha \exp (T x) e, \quad x \geqslant 0 .
$$

A distribution $G$ defined by (33) is called a phase-type distribution (PH distribution). The pair $(\alpha, T)$ is called the representation of $G$. If the Markov process is restarted instantaneously after each absorption into state $m+1$ and if each process restart is considered a renewal, then the interrenewal time distribution is the PH distribution (33). Such a process is called a phase-type renewal process and its infinitesimal generator is given by

$$
Q^{*}=T+\left(1-\alpha_{m+1}\right)^{-1} T^{0} \alpha
$$

A direct and very useful analogon of Lemma 2.2.2 in Neuts [13, pp. 45] is the following lemma.

Lemma 7. The probability distribution $P\left(Z_{t} \leqslant z\right)$ of the forward recurrence time, corresponding to the $P H$ distribution with representation $(\alpha, T)$ is given by

$$
P\left(Z_{t} \leqslant z\right)=1-\alpha \exp \left(Q^{*} t\right) \exp (T z) e, \quad t, z \geqslant 0 .
$$

Proof. The unconditional probabilities $w_{j}(t)$ that the PH-renewal process is in state $j$ at time $t$, $j=1, \ldots, m$, satisfy the system of differential equations

$$
w^{\prime}(t)=w(t) Q^{*}
$$

with initial conditions $w(0)=\alpha$. Its solution is given by

$$
\boldsymbol{w}(t)=\alpha \exp \left(Q^{*} t\right)
$$

Clearly, $P\left(Z_{t} \leqslant z\right)$ is a $\mathrm{PH}$ distribution with representation $(\boldsymbol{w}(t), T)$. 
Let $\psi(t, z)$ and $\psi(z)$ denote the probability density function of $Z_{t}$ and $Z$ respectively. The following statements are readily verified from Lemma 7 respectively (11):

$$
\begin{aligned}
& \psi(t, z)=\frac{\partial}{\partial z} \Psi(t, z)=\alpha \exp \left(Q^{*} t\right) \exp (T z) T^{0}, \\
& \mathrm{E} Z_{t}=-\alpha \exp \left(Q^{*} t\right) T^{-1} e, \\
& \psi(z)=\frac{P(Y>z)}{\mathrm{E} Y}, \quad \mathrm{E} Z=\frac{\mathrm{E} Y^{2}}{2 \mathrm{E} Y} .
\end{aligned}
$$

The distribution of the forward recurrence time of a $K_{2}$ distribution

With (34) an analytic expression for $\Psi(t, z)$ for the $\mathrm{K}_{2}$ distribution is easily obtained. For the $\mathrm{K}_{2}$ distribution we have

$$
T=\left(\begin{array}{cc}
-\lambda_{1} & (1-p) \lambda_{1} \\
0 & -\lambda_{2}
\end{array}\right), \quad T^{0}=\left(\begin{array}{c}
p \lambda_{1} \\
\lambda_{2}
\end{array}\right), \quad \alpha=(1,0)
$$

and the generator of the associated PH-renewal process is given by

$$
Q^{*}=\left(\begin{array}{cc}
-(1-p) \lambda_{1} & (1-p) \lambda_{1} \\
\lambda_{2} & -\lambda_{2}
\end{array}\right) .
$$

After some calculus we obtain

$$
w_{1}(t)=\frac{\lambda_{2}}{\lambda_{1}(1-p)+\lambda_{2}}-\frac{\lambda_{1}(1-p)}{\lambda_{1}(1-p)+\lambda_{2}} \mathrm{e}^{-\left\{\lambda_{1}(1-p)+\lambda_{2}\right\} t}
$$

and

$$
\begin{aligned}
& \exp (T z) \boldsymbol{e}=\left(\begin{array}{c}
\frac{p \lambda_{1}-\lambda_{2}}{\lambda_{1}-\lambda_{2}} \mathrm{e}^{-\lambda_{1} z}+\frac{(1-p) \lambda_{1}}{\lambda_{1}-\lambda_{2}} \mathrm{e}^{-\lambda_{2} z} \\
\mathrm{e}^{-\lambda_{2} z}
\end{array}\right), \quad \lambda_{1} \neq \lambda_{2}, \\
& \exp (T z) \boldsymbol{e}=\left(\begin{array}{c}
\mathrm{e}^{-\lambda z}+(1-p) \lambda z \mathrm{e}^{-\lambda z} \\
\mathrm{e}^{-\lambda z}
\end{array}\right), \quad \lambda_{1}=\lambda_{2}=\lambda .
\end{aligned}
$$

Now we can write

$$
\begin{aligned}
P\left(Z_{t} \leqslant z\right)= & 1-\left(w_{1}(t), 1-w_{1}(t)\right) \exp (T z) e \\
& = \begin{cases}1-w_{1}(t) \frac{p \lambda_{1}-\lambda_{2}}{\lambda_{1}-\lambda_{2}} \mathrm{e}^{-\lambda_{1} z}-\left[1-w_{1}(t) \frac{p \lambda_{1}-\lambda_{2}}{\lambda_{1}-\lambda_{2}}\right] \mathrm{e}^{-\lambda_{2} z}, & \lambda_{1} \neq \lambda_{2}, \\
1-e^{-\lambda z}-w_{1}(t)(1-p) \lambda z \mathrm{e}^{-\lambda z}, & \lambda_{1}=\lambda_{2}=\lambda .\end{cases}
\end{aligned}
$$

The probability density $\psi(t, z)$ and $\mathrm{E} Z$, are easily obtained from (38) (or directly from (35) resp. (36), using $\left.\alpha \exp \left(Q^{*} t\right)=\left(w_{1}(t), 1-w_{1}(t)\right)\right)$. Let $\psi(z)$ denote the probability density function of $Z$. In Figure 3 we plotted the probability densities $\psi(t, z)$ and $\psi(z)$ for $c_{Y}^{2}=\frac{1}{2}$ and $c_{Y}^{2}=2$.

Remark. For any $\mathrm{K}_{2}$ distribution the curve of $\psi(t, z)$ intersects $\psi(z)$ in the same point $z_{c}$ independently of $t$, which is given by

$$
z_{c}= \begin{cases}\frac{\log \left(\lambda_{1}\right)-\log \left(\lambda_{2}\right)}{\lambda_{1}-\lambda_{2}}, & \lambda_{1} \neq \lambda_{2}, \\ 1 / \lambda, & \lambda_{1}=\lambda_{2}=\lambda .\end{cases}
$$




\section{Acknowledgements}

The authors like to thank Marcel van der Lee and Theo Mandos for carrying out the simulation studies reported in this paper.

\section{References}

[1] Apostol, T.M., Mathematical Analysis: A Modern Approach to Advanced Calculus. Addison-Wesley Publishing Company, 1965.

[2] Bäckert. W., and Rippin. D.W.T., "The determination of maintenance strategies for plants subject to breakdown". Computers and Chemical Engineering 9 (1985) 113-126.

[3] Barlow. R.E., and Proschan. F., Mathematical Theory of Reliability, Wiley, New York, 1965.

[4] Berg. M.. "General trigger-off replacement procedures for two-unit systems", Naval Research Logistics Quarterly 25 (1978) $15-29$.

[5] Berg. M.. "A marginal cost analysis for preventive maintenance policies", European Journal of Operational Research, 4 (1980) $136-142$.

[6] Berg, M., and Epstein, B.. "A modified block replacement policy", Naval Research Logistics Quarterly 23 (1976) 15-24.

[7] Dekker. R.. and Dijkstra. M.C.. "Opportunity based age replacement", to appear in Naval Research Logistics.

[8] Duncan. J.. and Scholnick, L.S., "Interrupt and opportunistic replacement strategies for systems of deteriorating components", Operational Research Quarterly 24 (1973) 271-283.

[9] Giblin. M.T., "Derivation of renewal functions using discretisation", in: 8th Advances in Reliability Techniques Symposium, 1984.

[10] Hanscom. M.A., and Cleroux, R., "The block replacement problem", Journal of Statistical Computations and Simulations 3 (1975) $233-248$.

[11] Jorgensen, D.W., McCall, J.J., and Radner. R., Optimal Replacement Policy, North-Holland, Amsterdam, 1967.

[12] Liang. T.Y.. "Optimum piggyback preventive maintenance policies". IEEE Transactions on Reliability 34 (1985) $529-538$

[13] Neuts. M.F., Matrix-Geometric Solutions in Stochastic Models. Johns Hopkins University Press, Baltimore, MD, 1981.

[14] Pierskalla, W.P., and Voelker, J.A., "A survey of maintenance models: The control and surveillance of deteriorating systems", Naval Research Logistics Quarterly 23 (1976) 353-388.

[15] Ross, S.M., Applied Probability Models with Optimization Applications, Holden-Day, San Fransisco, 1970.

[16] Sethi, D.P.S.. "Opportunistic Replacement Policies", in I.N. Shimi \& C.P. Tsokos (eds.), The Theory and Applications of Reliability, Vol. 1, Academic Press, New York, 1977, 433-447.

[17] Sherif. Y.S., and Smith, M.L., "Optimal maintenance models for systems subject to failure - a review", Naval Research Logistics Quarterly 28 (1981) 47-74

[18] Sherif, Y.S.. "Reliability analyses. Optimal inspection and maintenance schedules for failing systems", Microelectronics Reliability 22 (1982) 59-115.

[19] Smeitink. E.. and Dekker, R., "A simple approximation to the renewal function", IEEE Transactions on Reliability 39 (1990) $71-75$.

[20] Stroud, A.H., and Secrest. D., Gaussian Quadrature Formulas, Prentice-Hall, Englewood Cliffs (1966).

[21] Tijms, H.C.. Stochastic Modelling and Analysis: A Computational Approach, John Wiley, New York, 1986.

[22] Woodman, R.C., "Replacement policies for components that deteriorate", Operational Research Quarterly 18 (1967) 267-280. 